\title{
Association Between Prevalence of Depression and Anxiety in Cystic Fibrosis Patients and Their Parents: A Cross-sectional Study in Iran
}

\author{
Azam Orooji (iD ${ }^{1}$, Ali Valinejadi (iD ${ }^{2}$, Maryam Hassanzad (iD ${ }^{3,},{ }^{*}$, Mohammadreza Boloursaz (iD ${ }^{3}$ and Ali \\ Akbar Velayati (iD ${ }^{4}$ \\ ${ }^{1}$ Department of Advanced Technologies, School of Medicine, North Khorasan University of Medical Sciences, Bojnurd, Iran \\ ${ }^{2}$ Social Determinants of Health Research Center, Semnan University of Medical Sciences, Semnan, Iran \\ ${ }^{3}$ Pediatric Respiratory Diseases Research Center, National Research Institute of Tuberculosis and Lung Diseases (NRITLD), Shahid Beheshti University of Medical Sciences, \\ Tehran, Iran \\ ${ }^{4}$ Mycobacteriology Research Center, National Research Institute of Tuberculosis and Lung Diseases (NRITLD), Shahid Beheshti University of Medical Sciences, Tehran, Iran \\ "Corresponding author: Pediatric Respiratory Diseases Research Center, National Research Institute of Tuberculosis and Lung Diseases (NRITLD), Shahid Beheshti University of \\ Medical Sciences, Tehran, Iran. Email: mar_hassanzad@yahoo.com
}

Received 2021 February 08; Accepted 2021 February 20.

\begin{abstract}
Background: Patients with chronic diseases and their caregivers are at risk for mental disorders, such as depression and anxiety. Objectives: This study aimed to investigate the prevalence of depression and anxiety symptoms among cystic fibrosis (CF) patients and their parent caregivers in Iran.

Methods: This descriptive-analytical cross-sectional study was carried out on 96 participants, including 60 patients and 36 parents. The Hospital Anxiety and Depression Scale was used to measure the depression and anxiety in this study. The research site was the Pediatric Respiratory Diseases Research Center of the National Research Institute of Tuberculosis and Lung Diseases in Tehran, Iran. SPSS software (version 22) was used to examine and analyze the raw data.

Results: The prevalence of anxiety in patients with a mean incidence of $12.17 \%$ was higher than that in parents with a mean incidence of $11.81 \%$. Moreover, the prevalence of depression in patients and parents (10.58\%) was almost similar. In the patients' group, there was a direct and significant relationship between the duration of diagnosis with depression and anxiety; nevertheless, there was an inverse and significant relationship between the patient's age and anxiety. In the parents' group, there was a significant inverse association between age and depression, as well as between the duration of diagnosis and anxiety. However, there was no significant relationship between anxiety and depression, neither in the parents' group nor in the patients' group.

Conclusions: The high prevalence of depression and anxiety symptoms among CF patients and their parents indicates the poor mental state of these individuals, which requires planning and implementation of psychological interventions for this group.
\end{abstract}

Keywords: Cystic Fibrosis, Depression, Anxiety

\section{Background}

A chronic underlying disease is one of the most important risk factors for mental disorders, such as anxiety and depression (1). Chronic diseases affect the patient's daily activities and impose too much stress on the patient and his/her family (2). In general, individuals with chronic diseases are $41 \%$ more likely than others to have psychiatric disorders (1). Research studies, meta-analyses, and systematic reviews demonstrate that adults and children with chronic diseases and their families are more at risk for depression and anxiety than others (3).

Cystic fibrosis (CF) is an autosomal recessive disorder resulting from a deviation in a gene encoding the cystic fibrosis transmembrane conductance regulator protein.
CF causes abnormal ion transport throughout the body. This disease causes problems with mucus secretion in the lungs, leading to chronic lung infection and inflammation (4). The CF is a chronic incurable genetic disorder threatening individuals' lives. It is estimated that one in two to three thousand white neonates has CF (5).

Recent advances in CF management and treatment have led to an increase in life expectancy among CF patients. In most countries, the mean age of survival for $\mathrm{CF}$ patients is 30 to 40 years, representing a 10-year increase, compared to that reported for the previous decade $(6,7)$. Furthermore, this has caused CF patients and their families to face many problems by increasing the patient's age, such as increasing the symptom burden and number of complications, indicating that these individuals need 
more time-consuming and complex treatment processes (6). Therefore, it is important to pay attention to the mental problems of CF patients because studies showed that depressed patients are more likely to miss physician's visits, have less life expectancy, and do not pursue their treatment. Therefore, the early diagnosis and treatment of depression and other mental health problems can improve the long-term consequences of $\mathrm{CF}(6)$.

Studies proved that the prevalence of depression in $\mathrm{CF}$ patients is $8-29 \%$ for children and adolescents and 13 $33 \%$ for adults $(8,9)$. The prevalence of anxiety in adults is within the range of $30-33 \%(10)$. Mortality and complications of the disease pose many cognitive, emotional, and behavioral challenges not only for CF children but also for their families (4). The prevalence of depression among these caregivers is within the range of 20 - 35\% (11). Moreover, the International Depression Epidemiological Study (TIDES) holds forth that adolescents with CF whose parents suffer from depression or anxiety are twice as likely to be depressed and anxious (12). Depression, anxiety, and insanity in CF patients not only affect their family members but also, in the short and long term, can affect the severity of the disease and its complications (6).

Previous studies in this regard can be divided into two groups. The first group includes the studies separately examining the patients or patients' parents. The second group includes the studies considering both patients and parents, investigating the relationship between the mental health of both groups.

The association between anxiety and depression with the quality of life in CF patients has been considered in a study by Yohannes et al. (5). The aforementioned study was performed on $121 \mathrm{CF}$ patients over 18 years of age. A study conducted by Havermans et al. (11) also examined the relationship between anxiety, depression, and clinical symptoms with the quality of life in CF patients. The aforementioned study was conducted on 57 adult CF patients with a mean age of 26.7 years. Another survey (13) in Spain studied the relationship between the quality of life with depression and anxiety. The study population was considered 336 CF patients. Yılmaz et al. (14) investigated sleep disorders, anxiety, and depression among mothers whose children had CF or asthma. The aforementioned study was conducted on a sample, including mothers of 62 children with asthma, 21 children with $\mathrm{CF}$, and 35 healthy children.

As previously reported, the second group of studies considered both patients and parents. The largest and most comprehensive study in this regard (i.e., the TIDES) observed nine countries in terms of depression prevalence among CF patients and their parents. The countries included in the study were Belgium, Germany, Italy, Spain, Sweden, the Netherlands, Turkey, the United Kingdom, and the United States. In the aforementioned study, $6088 \mathrm{CF}$ patients over 12 years of age and 4102 parents of children with CF under 18 years of age were studied from $154 \mathrm{CF}$ centers in Europe and the United States (12). Additionally, Catastini et al. (2) studied the prevalence of depression and anxiety in CF patients and their parents and examined their association with the clinical signs of the disease. The aforementioned study was performed on $614 \mathrm{CF}$ patients and 443 parents in Italy.

To date, in Iran, no studies have specifically studied the psychological problems of CF patients. However, several studies are related to respiratory and non-respiratory diseases, all of which belong to the first group of studies. The end-stage of kidney disease and dialysis severely affect the physical and mental health of patients. Due to the long duration of treatment and lifestyle changes in these patients, a study (15) was conducted to determine the prevalence of depression in patients undergoing hemodialysis in Birjand, Iran. The results of studies indicated a high prevalence of psychological complications in patients with multiple sclerosis (MS). Therefore, a study was designed to investigate the prevalence of personality disorders in MS patients in South Khorasan province, Iran. The aforementioned study was performed on 55 MS patients (16).

In a study conducted on respiratory patients, Vagharfard (17) focused on tuberculosis because it was particularly prevalent among individuals with mood disorders, such as depression. For this reason, physicians should also be aware of the clinical manifestations of depression when treating tuberculosis patients, as this may lead to treatment discontinuation or disordered treatment. The study by Vagharfard was performed on 76 tuberculosis patients. In various studies, Safa et al. and Fallah Tafti et al. investigated the prevalence of depression and anxiety, quality of life, and sleep quality among asthmatic children (18-20), chronic obstructive pulmonary disease children (21, 22), mothers of asthmatic children (23), and mothers of children with asthma or CF (24). However, as previously mentioned, none of the studies in Iran has considered both groups of patients and parents simultaneously and studies the relationship between the psychological problems of both groups. Table 1 shows the used questionnaires, year of article publication, and country of study.

Due to the high prevalence of anxiety and depression in CF patients, it is recommended that these patients should be evaluated for depression and anxiety once a year and referred to a psychologist if necessary (1). There are two main methods for the assessments of depressive symptoms, namely diagnostic interviews and standardized screening measures. The diagnostic interview is a golden standard to diagnose depression; however, it takes time and requires an expert, such as a psychologist or psy- 


\begin{tabular}{|c|c|c|c|c|}
\hline Country & Target Group & Questionnaire & Year of Publication & Author (s) \\
\hline Nine countries & CF patients and their parents & HADS, CES-D & 2014 & Quittner et al. (12) \\
\hline Italy & CF patients and their parents & HADS, CES-D & 2016 & Catastini et al. (2) \\
\hline England & CF patients & $\begin{array}{l}\text { HADS, Cystic Fibrosis Quality of Life } \\
\text { Questionnaire }\end{array}$ & 2012 & Yohannes et al. (5) \\
\hline Turkey & Mothers of patients with CF or asthma & HADS, PSQI & 2008 & Yllmaz et al. (14) \\
\hline Belgium & CF patients & HADS, CFQ-14+ & 2008 & Havermans et al. (11) \\
\hline Spain & CF patients & HADS, CFQ-14+ & 2016 & Olveira et al. (13) \\
\hline Iran & Hemodialysis patients & Beck Depression Inventory & 2010 & Mogharab et al. (15) \\
\hline Iran & Multiple sclerosis patients & Beck Depression Inventory & 2015 & Javidan (16) \\
\hline Iran & Tuberculosis patients & Beck Depression Inventory & 2014 & Vagharfard (17) \\
\hline Iran & Asthmatic patients & GHQ-28 & 2014 & Safa et al. (18) \\
\hline Iran & Asthmatic patients & HADS & 2013 & Fallah Tafti et al. (19) \\
\hline Iran & Asthmatic patients & $\begin{array}{l}\text { St. George's Respiratory Questionnaire, } \\
\text { GHQ-28 }\end{array}$ & 2011 & Fallah Tafti et al. (20) \\
\hline Iran & COPD patients & PSQI, Patient Health Questionnaire & 2017 & Eslaminejad et al. (21) \\
\hline Iran & COPD patients & Hamilton Anxiety Scale & 2015 & Safa et al. (22) \\
\hline Iran & Mothers of asthmatic patients & Symptom Checklist-90-Revised & 2014 & Safa and Ghasem Boroujerdi (23) \\
\hline Iran & Mothers of patients with CF or asthma & PSQI, HADS & 2012 & Safa et al. (24) \\
\hline
\end{tabular}

chiatrist, specifically trained in this field. On the contrary, screening criteria are concise and inexpensive and do not require special training. These criteria have been attentively evaluated by psychometrics and are entirely relevant to diagnostic interviews and appropriate for clinical centers or research protocols (25).

Considering the importance of the issue, the present study investigated the prevalence of depression and anxiety disorders in CF patients and their parents in Iran. In this study, the second method of measuring depression and anxiety symptoms, namely using the screening criteria, was used. Moreover, since the Pediatric Respiratory Diseases Research Center of National Research Institute of Tuberculosis and Lung Diseases (NRITLD) is a referral center for CF patients in Iran, it was selected as the research site.

\section{Objectives}

This survey answers the following questions: (1) what is the prevalence of depression among CF patients and their parents; (2) what is the prevalence of anxiety among CF patients and their parents; (3) is there any association between depression and anxiety in CF patients and their parents?

\section{Methods}

This descriptive-analytical cross-sectional study was conducted at the Pediatric Respiratory Diseases Research
Center of NRITLD as a referral center for CF patients in Iran in 2020. In this study, 110 questionnaires were distributed among all CF patients who were hospitalized within January to May 2020 and their parents. All the questionnaires were collected anonymously. Furthermore, consent forms were signed by the subjects before participating in the study. The questionnaire consisted of two sections. The first section included demographic data, namely age, gender, educational level, and duration of disease diagnosis. In the second section, the Hospital Anxiety and Depression Scale (HADS) (26) was used as a tool to measure depression and anxiety. The validity and reliability of the Persian version of the questionnaire have been investigated in previous studies (27).

The HADS questionnaire is a 14-item screening scale based on the patient's self-report, including a 7-item scale for measuring depression (HADS-D) and a 7-item scale for measuring anxiety (HADS-A). For each item, there are four possible answers with a score of 0 to 3 . Moreover, the maximum possible score for each of the subscales of depression and anxiety is 21 . A higher score on each subscale indicates more severe symptoms of depression or anxiety. A score of 7 was considered a depression and anxiety threshold in each subscale. For both subscales of depression and anxiety, the scores within the ranges of $0-7,8-10$, and 11 - 15 were considered normal, mild, and moderate, respectively. Furthermore, scores higher than 16 were regarded as severe (12).

Descriptive and inferential statistics were used to ana- 
lyze the research data. Descriptive statistics were used to describe demographic data and calculate frequencies and scattering indices, such as mean and standard deviation. The Kolmogorov-Smirnov test, independent $t$-test, Pearson and Spearman correlation coefficients, and Mann-Whitney $\mathrm{U}$ test were used for inferential statistics. In addition, SPSS software (version 22) was used to examine and analyze the raw data.

\section{Results}

Out of the 110 distributed questionnaires, 101 cases were filled out and returned (response rate: 91.8\%). After excluding the confounding questionnaires, 96 filled out questionnaires (36 for parents and 60 for patients) were analyzed in this study. Tables 2 and 3 show the demographic data and duration of diagnosis for both groups of patients and parents, respectively.

Table 2 tabulates that most patients are male, and the highest frequency is observed in the age group of 7-18 years and educational level of high school. In addition, the duration of diagnosis for most patients was 10 - 19 years. However, Table 3 shows that mothers participate in the study more than fathers. The age group of 40 - 59 years and educational level of elementary school had the highest frequency among the parents. Moreover, in half of the cases, the duration of diagnosis was 10 - 19 years. In the data preprocessing stage, the missing items were completed with the mean value (for the quantitative data) or mode value (for the qualitative data). The details of the scores for the participants' depression and anxiety scales are shown in Table 4.

Table 4 shows that the prevalence of anxiety in patients with a mean of $12.17 \%$ is higher than that reported for parents with a mean of $11.81 \%$. Furthermore, $75 \%$ of the patients and $63.9 \%$ of the parents were in moderate to severe anxiety levels, and the prevalence of depression in patients and parents (10.58\%) was almost equal. Severe depression was reported for either parents or patients; nevertheless, 56.7\% of the patients and $55.6 \%$ of the parents had moderate depression. The results demonstrated that the prevalence of depression and anxiety was higher among fathers than mothers. In addition, the prevalence of anxiety was higher among male patients than female patients; however, the prevalence of depression was higher among female patients than male patients. The use of the Mann-Whitney $\mathrm{U}$ test showed that none of these differences was statistically significant $(\mathrm{P}>0.05)$. The Kolmogorov-Smirnov test showed that the significance level for all the variables was higher than 0.05; therefore, the distribution of all the sample variables in both groups of patients and parents was normal. Then, the association between variables was investigated using Pearson and Spearman correlation coefficients. Table 5 tabulates the correlation coefficients and significance levels.

Table 5 shows that in the patients' group, there is a direct and significant relationship between the duration of diagnosis with depression and anxiety. Moreover, there was an inverse and significant relationship between the patient's age and anxiety. In the parents' group, there was a significant inverse association between age and depression, as well as between the duration of diagnosis and anxiety. According to the findings, there was no significant relationship between anxiety and depression in either parents' or patients' groups. In addition, the independent $t$ test revealed that there was no significant difference $(\mathrm{P}=$ 0.301 and $P=0.211$ ) between the mean of depression and anxiety in both groups of parents and patients.

\section{Discussion}

This survey has been the first study to investigate the prevalence of anxiety and depression among CF patients and their parents in Iran. According to the findings, $75 \%$ of the patients and $63.9 \%$ of the parents had moderate to severe anxiety. Although severe depression was not reported in either parents or patients, 56.7\% of the patients and $55.6 \%$ of the parents had moderate depression. In the TIDES study (12), depressive symptoms (the HADS score of above 7) were observed in $10 \%$ of adolescents, $19 \%$ of adults, $37 \%$ of mothers, and $31 \%$ of fathers. Furthermore, there were anxiety symptoms (the HADS score of above 7) in $22 \%$ of adolescents, $32 \%$ of adults, $48 \%$ of mothers, and $36 \%$ of fathers.

Catastini et al. (2) proved that $14 \%$ of patients and $19.9 \%$ of parent caregivers were anxious, and the prevalence rates of depression in patients, mothers, and fathers were 5.7, 8.7 , and $2.8 \%$, respectively. In the aforementioned study, HADS scores of above 10 were considered the measure of depression or anxiety, and patients were over 11 years of age. The prevalence of 33\% for anxiety symptoms and $17 \%$ for depressive symptoms in another study (5) was reported in patients with a mean age of 30 years. The HADS score of above 8 was considered for having symptoms. In a study performed by Havermans et al. (11), the HADS score was above 7 in 30 and 13\% of patients for anxiety and depression, respectively. The mean age of patients in the aforementioned study was 26.79 years.

In a study (13) conducted on patients over 18 years of age, 12.2 and $29.7 \%$ of subjects had depressive and anxiety symptoms, respectively, in which the HADS score of above 8 was considered the threshold. The prevalence of depression and anxiety symptoms in another study performed on patients over 18 years of age was reported to be $45 \%$ (28). 


\begin{tabular}{|c|c|c|c|c|}
\hline Values & No. & $\%$ & Mean & SD \\
\hline Age $(y)$ & & & 18.18 & 6.43 \\
\hline$<7$ & 1 & 1.7 & & \\
\hline 7-18 & 32 & 53.3 & & \\
\hline$>18$ & 27 & 45 & & \\
\hline Gender & & & - & - \\
\hline Female & 22 & 36.7 & & \\
\hline Male & 38 & 63.3 & & \\
\hline Educational level & & & - & - \\
\hline Preschool & 1 & 1.7 & & \\
\hline Elementary school & 15 & 25 & & \\
\hline Junior high school & 12 & 20 & & \\
\hline High school & 20 & 33.3 & & \\
\hline Bachelor of science & 11 & 18.3 & & \\
\hline Missing & 1 & 1.7 & & \\
\hline Duration of cystic fibrosis diagnosis $(y)$ & & & 11.52 & 6.21 \\
\hline$<10$ & 25 & 41.7 & & \\
\hline $10-19$ & 26 & 43.3 & & \\
\hline$>20$ & 8 & 13.3 & & \\
\hline Missing & 1 & 1.7 & & \\
\hline
\end{tabular}

\begin{tabular}{|c|c|c|c|c|}
\hline Values & No & $\%$ & Mean & SD \\
\hline Age $(y)$ & & & 44.21 & 6.10 \\
\hline$<20$ & 0 & 0 & & \\
\hline $20-39$ & 8 & 22.2 & & \\
\hline $40-59$ & 20 & 55.6 & & \\
\hline$\geq 60$ & 0 & 0 & & \\
\hline Missing & 8 & 22.2 & & \\
\hline Gender & & & - & - \\
\hline Female & 30 & 83.3 & & \\
\hline Male & 6 & 16.7 & & \\
\hline Educational level & & & - & - \\
\hline Preschool & 2 & 5.6 & & \\
\hline Elementary school & 14 & 38.9 & & \\
\hline Junior high school & 11 & 30.5 & & \\
\hline High school & 8 & 22.2 & & \\
\hline Bachelor of science & 0 & 0 & & \\
\hline Master of science & 0 & 0 & & \\
\hline $\mathrm{PhD}$ & 0 & 0 & & \\
\hline Missing & 1 & 2.8 & & \\
\hline Duration of cystic fibrosis diagnosis (y) & & & 10.56 & 5.53 \\
\hline$<10$ & 17 & 47.2 & & \\
\hline $10-19$ & 18 & 50 & & \\
\hline$\geq 20$ & 1 & 2.8 & & \\
\hline
\end{tabular}

Furthermore, in a study by Cronly et al. performed on patients over 17 years of age, 26.4 and 9\% of patients had the HADS score of above 7 for anxiety and depression, respectively (29). In another study conducted by Cronly et al. on the parents of children under 17 years of age, it was shown that 38 and $12 \%$ of subjects had the HADS score of above 7 for anxiety and depression, respectively (30).

The present study suggested that gender did not make any significant difference between the prevalence of depression and anxiety. Some studies confirmed the findings of the current study $(2,13)$, and some studies contradicted the findings of the current study and reported a higher prevalence of depression and anxiety among women than men in both patients and parents $(2,28,30)$. The current study also suggested that age was inversely related to anxiety in patients and depression in parents. Some previous 


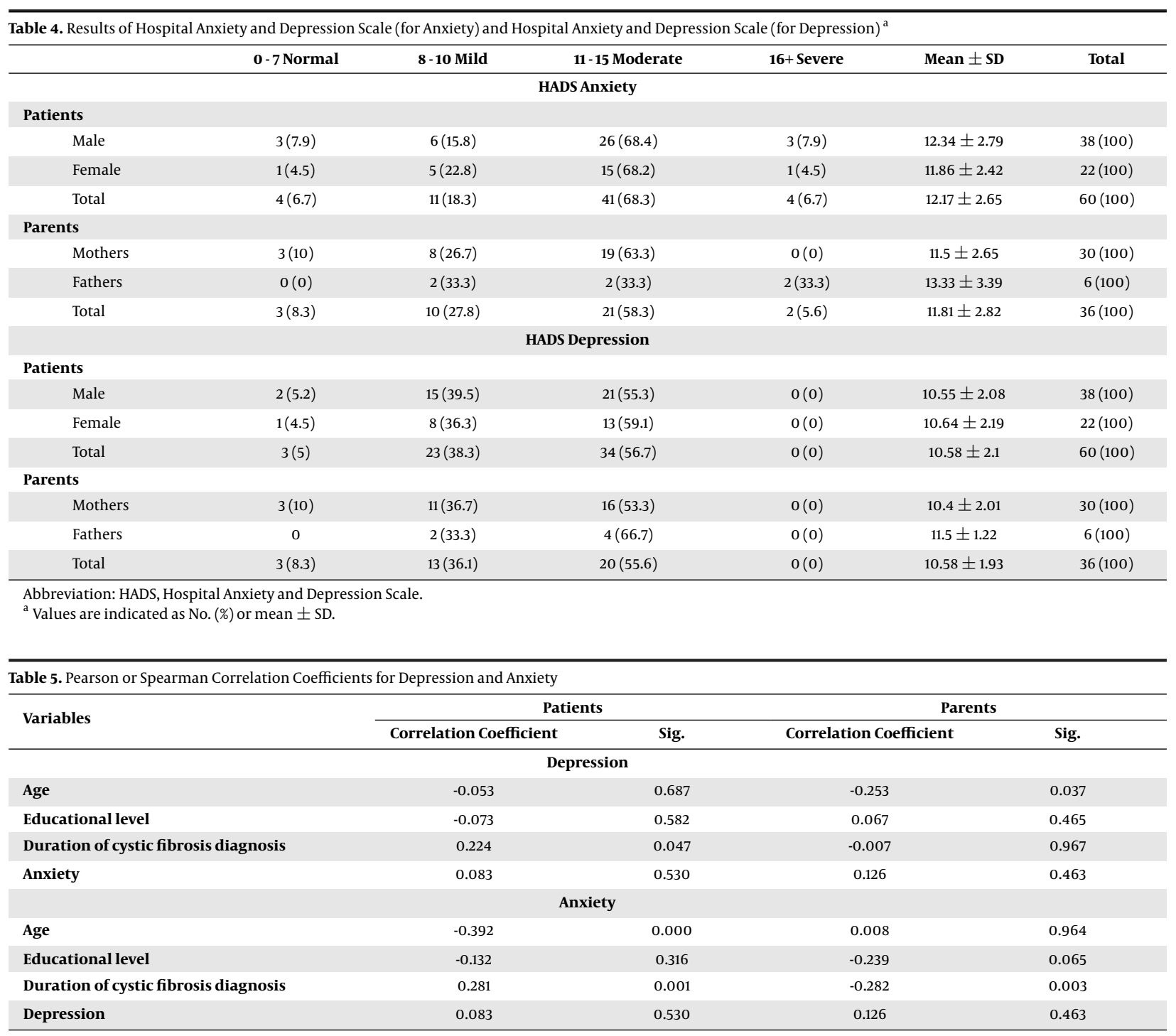

studies reported a direct association between the age of patients or parents and prevalence of depression and anxiety (5). Moreover, some studies observed no association in this regard $(13,30)$. Nonetheless, some studies have confirmed an inverse relationship between patient's age and their anxiety (2). In this study, the educational level had no effect on depression and anxiety in either group of parents or patients; however, other studies reported an inverse association between educational level with depression and anxiety prevalence $(13,28)$.

In the present study, the duration of diagnosis was considered an indicator of the patient's current state of life. The findings suggested a direct and significant relationship between the duration of diagnosis with depression and anxiety in the patients' group; nonetheless, in the parents' group, there was a significant inverse relationship between the duration of diagnosis and anxiety. However, some studies did not find any significant relationship between parents' depression and anxiety with their children's duration of diagnosis (30). Numerous studies inferred an increase in anxiety and depression among patients and parent caregivers in case of the exacerbation of the patient's respiratory condition $(5,30)$; nevertheless, some other studies concluded the opposite $(13,28)$. The results of the present study indicated that there was no significant relationship between the prevalence of depression and anxiety in either parents' or patients' groups; however, a direct relationship was concluded in this regard in some studies $(11,13)$. 


\subsection{Conclusion}

In the present study, due to the small sample size, it was not possible to separate the age groups; nevertheless, by comparing the findings of this study to the findings of previous studies, it was observed that regardless of demographic characteristics, anxiety and depression in CF patients and parent caregivers had a high prevalence in Iran. Therefore, it is necessary to pay close attention to the mental health status of these individuals. Therefore, it is of paramount importance to plan for regular monitoring of these individuals' mental health and provide mental health services to reduce the levels of depression and anxiety.

\section{Acknowledgments}

The authors would like to express their gratitude to patients and their parents participating in the current study.

\section{Footnotes}

Authors' Contribution: Study concept and design, Azam Orooji and Maryam Hssanzad; Acquisition of the data, Ali Valinejadi, Maryam Hssanzad, and Mohammad Reza Boloursaz; Analysis and interpretation of the data, Azam Orooji and Ali Valinejadi; Drafting of the manuscript, Azam Orooji, Ali Valinejadi, and Maryam Hssanzad; Critical revision, Maryam Hssanzad, Mohammad Reza Boloursaz, and Ali Akbar Velayati; All the authors approved the final version of the paper.

Conflict of Interests: The authors declare that there is no conflict of interest.

Ethical Approval: This study was approved by the Ethics Committee of Shahid Beheshti University of Medical Sciences (IR.SBMU.NRITLD.REC.1397.040).

Funding/Support: This study was supported by a grant (No.: 14871) from Shahid Beheshti University of Medical Sciences, Tehran, Iran.

Informed Consent: Written consent was obtained from all the patients and their parents.

\section{References}

1. Cruz I, Marciel KK, Quittner AL, Schechter MS. Anxiety and depression in cystic fibrosis. Semin respir crit care med. 2009;30(5):569-78. doi: 10.1055/s-0029-1238915. [PubMed: 19760544].

2. Catastini P, Di Marco S, Furriolo M, Genovese C, Grande A, Iacinti E, et al. The prevalence of anxiety and depression in Italian patients with cystic fibrosis and their caregivers. Pediatr Pulmonol.2016;51(12):1311-9. doi: 10.1002/ppul.23566. [PubMed: 27759954].
3. Quittner AL, Abbott J, Georgiopoulos AM, Goldbeck L, Smith B, Hempstead SE, et al. International Committee on Mental Health in cystic fibrosis: Cystic fibrosis foundation and european cystic fibrosis society consensus statements for screening and treating depression and anxiety. Thorax. 2016;71(1):26-34. doi: 10.1136/thoraxjnl-2015-207488. [PubMed: 26452630]. [PubMed Central: PMC4717439].

4. Ernst MM, Johnson MC, Stark LJ. Developmental and psychosocial issues in cystic fibrosis. Child Adolesc Psychiatr Clin NAm. 2010;19(2):26383. viii. doi: 10.1016/j.chc.2010.01.004. [PubMed: 20478499]. [PubMed Central: PMC2874200].

5. Yohannes AM, Willgoss TG, Fatoye FA, Dip MD, Webb K. Relationship between anxiety, depression, and quality of life in adult patients with cystic fibrosis. Respir Care. 2012;57(4):550-6. doi: 10.4187/respcare.01328. [PubMed: 22004950].

6. Sawicki GS, Tiddens H. Managing treatment complexity in cystic fibrosis: Challenges and opportunities. Pediatr Pulmonol. 2012;47(6):523-33. doi: 10.1002/ppul.22546. [PubMed: 22467341].

7. Havermans T, Colpaert K, Vanharen L, Dupont LJ. Health related quality of life in cystic fibrosis: To work or not to work? J Cyst Fibros. 2009;8(3):218-23. doi: 10.1016/j.jcf.2009.03.002. [PubMed:19328745].

8. Smith BA, Modi AC, Quittner AL, Wood BL. Depressive symptoms in children with cystic fibrosis and parents and its effects on adherence to airway clearance. Pediatr Pulmonol. 2010;45(8):756-63. doi: 10.1002/ppul.21238. [PubMed: 20597082].

9. Latchford G, Duff AJ. Screening for depression in a single CF centre. J Cyst Fibros. 2013;12(6):794-6. doi: 10.1016/j.jcf.2013.04.002. [PubMed: 23628513]

10. Fitz Simmons S. Patient registry. 2012 Annual data report. Bethesda, MD, USA: Cystic Fibrosis Foundation; 2012.

11. Havermans T, Colpaert K, Dupont LJ. Quality of life in patients with cystic fibrosis: Association with anxiety and depression. J Cyst Fibros. 2008;7(6):581-4. doi:10.1016/j.jcf.2008.05.010. [PubMed: 18692444].

12. Quittner AL, Goldbeck L, Abbott J, Duff A, Lambrecht P, Sole A, et al. Prevalence of depression and anxiety in patients with cystic fibrosis and parent caregivers: Results of The International Depression Epidemiological Study across nine countries. Thorax. 2014;69(12):1090-7. doi: 10.1136/thoraxjnl-2014-205983. [PubMed: 25246663].

13. Olveira C, Sole A, Giron RM, Quintana-Gallego E, Mondejar P, Baranda F, et al. Depression and anxiety symptoms in Spanish adult patients with cystic fibrosis: associations with healthrelated quality of life. Gen Hosp Psychiatry. 2016;40:39-46. doi: 10.1016/j.genhosppsych.2016.02.002. [PubMed: 26971246].

14. Yilmaz O, Sogut A, Gulle S, Can D, Ertan P, Yuksel H. Sleep quality and depression-anxiety in mothers of children with two chronic respiratory diseases: Asthma and cystic fibrosis. J Cyst Fibros. 2008;7(6):495500. doi:10.1016/j.jcf.2008.05.002. [PubMed:18585104].

15. Mogharab M, Hedayati H, Najafi R, Safari M, Amiri S, Adhami SH. [Prevalence of depression and life events in hemodialysis patients in Vali-Asr hospital in Birjand (2010)]. Mod Care J. 2011;8(3). Persian.

16. Javidan F. [A survey on prevalence of psychiatric disorders in MS patients in south Khorasan (1391) [dissertation]]. Birjand, Iran: Birjand University of Medical Sciences; 2016 . Persian.

17. Vagharfard J. [Prevalence of depression in Tuberculosis patients refered to Bandar Abbas health center [dissertation]]. Hormozgan, Iran: Hormozgan University of Medical Sciences; 2014. Persian.

18. Safa M, Mehrian P, Hassan Zad M. Prevalence of depression in children with asthma. J Compr Ped. 2014;5(2). e17327. doi: 10.17795/compreped17327.

19. Fallah Tafti S, Safa M, Talischi F, Ghassem Boroujerdi F. Evaluation of anxiety and depression in patients with asthma at Massih Daneshvari Hospital, Tehran. Sri Lanka J Psychiatry. 2013;4(1):7. doi: 10.4038/sljpsyc.v4i1.5716.

20. Fallah Tafti S, Cheraghvandi A, Safa M, Eragh DF, Mokri B, Talischi F. Study of depressed mood and quality of life in asthma patients in Tehran using the 28-item general health questionnaire. East Mediterr Health J. 2011;17(11):838-42. doi: 10.26719/2011.17.11.838. [PubMed: 22276491]. 
21. Eslaminejad A, Safa M, Ghassem Boroujerdi F, Hajizadeh F, Pashm Foroush M. Relationship between sleep quality and mental health according to demographics of 850 patients with chronic obstructive pulmonary disease. J Health Psychol. 2017;22(12):1603-13. doi: 10.1177/1359105316684937. [PubMed: 28770626].

22. Safa M, Fallah Tafti S, Talischi F, Ghassem Boroujerdi F. Severity of anxiety disorders in patients with chronic obstructive pulmonary disease.Iran JPsychiatry. 2015;10(2):128-32. [PubMed: 26884790].[PubMed Central: PMC4752526].

23. Safa M, Ghasem Boroujerdi F. Psychiatric problems in mothers of asthmatic children. J Compr Ped. 2014;5(1). e17086. doi: 10.17795/compreped-17086.

24. Safa M, Khalilzadeh S, Talischi F, Alizadeh S. Correlation of anxietydepression and sleep quality in mothers of children with cystic fibrosis and asthma. Tanaffos. 2012;11(1):44-8. [PubMed: 25191400]. [PubMed Central: PMC4153183].

25. Quittner AL, Barker DH, Snell C, Grimley ME, Marciel K, Cruz I. Prevalence and impact of depression in cystic fibrosis. Curr Opin Pulm Med. 2008;14(6):582-8. doi: 10.1097/MCP.ob013e3283121cfi. [PubMed: 18812836].
26. Zigmond AS, Snaith RP. The hospital anxiety and depression scale. Acta Psychiatr Scand. 1983;67(6):361-70. doi: 10.1111/j.16000447.1983.tb09716.x. [PubMed: 6880820].

27. Kaviani H, Seyfourian H, Sharifi V, Ebrahimkhani N. Reliability and validity of anxiety and depression hospital scales (HADS): Iranian patients with anxiety and depression disorders. Tehran Univ Med J. 2009;67(5):379-85.

28. Gersten R, Cannon B, Bowen M, Davies E, Brown AW. Evaluation of depression and anxiety and their influence on outcomes in adult patients with cystic fibrosis. C39. Identifying and Treating Anxiety and Depression in Pulmonary Diseases. New York, USA: American Thoracic Society; 2018. A4887 p.

29. Cronly J, Duff A, Riekert K, Horgan A, Lehane E, Perry I, et al Positive mental health and wellbeing in adults with cystic fibrosis: A cross sectional study. J Psychosom Res. 2019;116:125-30. doi: 10.1016/j.jpsychores.2018.11.016. [PubMed: 30579560].

30. Cronly J, Horgan AM, Lehane E, Howe B, Duff AJ, Riekert KA, et al. Anxiety and depression in parent caregivers of children with cystic fibrosis. J Child Fam Stud. 2019;28(5):1304-12. doi: 10.1007/s10826-019-013490 . 